\title{
Efficacy of Aflibercept Treatment and Its Effect on the Retinal Perfusion in the Oxygen-Induced Retinopathy Mouse Model of Retinopathy of Prematurity
}

\author{
Sarina M. Amin Andres Gonzalez Jade Guevara Charlotte Bolch \\ Lorick Andersen W. Clay Smith Swati Agarwal-Sinha \\ Department of Ophthalmology, University of Florida College of Medicine, Gainesville, IL, USA
}

\section{Keywords}

Anti-vascular endothelial growth factor · Retinopathy of prematurity · Aflibercept · Oxygen-induced retinopathy mouse model

\begin{abstract}
Introduction: Bevacizumab and ranibizumab, which are anti-vascular endothelial growth factor (VEGF) medications, are used frequently in the treatment for retinopathy of prematurity (ROP) in infants. Aflibercept, or VEGF Trap, has been used anecdotally, but translation and clinical studies are lacking. Objective: This study investigates the efficacy of aflibercept at reducing areas of non-perfused retina and studies its effect on normal angiogenesis in the oxygen-induced retinopathy mouse model of ROP. Methods: C57BL/6 $\mathrm{J}$ mice were assigned to room air control ( $n=21$ eyes) or hyperoxia with $75 \%$ oxygen ( $n=84$ eyes). The hyperoxic mice were assigned to 1 of 3 groups: 0 ng ( $n=14$ eyes), 100 ng ( $n=35$ eyes), or 1,000 ng ( $n=35$ eyes) of intravitreal aflibercept administered on postnatal day 14. Eyes were enucleated at PN17 and PN25 postinjection. Retinas were stained with anti-collagen IV antibody and photographed with microscopy. Areas of perfused and non-perfused retina were quantified using ImageJ software. Statistical com-
\end{abstract}

parisons were made using ANOVA with Tukey post hoc comparisons. Results: At PN17, there was no significant difference in the area of non-perfused retina between the hyperoxic control and the 100 and 1,000 ng aflibercept groups. At PN25, the $100 \mathrm{ng}(p<0.05)$ and $1,000 \mathrm{ng}(p=0.008)$ treatment groups displayed less non-perfusion compared to hyperoxic controls. At the 1,000 ng dose, there was increased non-perfusion compared to the $100 \mathrm{ng}$ dose ( $p=0.02$ ). There was reduced non-perfusion by PN25 compared to PN17 for the $100 \mathrm{ng}$ group ( $p<0.05$ ), with no difference in the 1,000 ng group. Conclusions: The study shows that the area of non-perfused retina decreases effectively with aflibercept at PN25 with $100 \mathrm{ng}$ dosage. With the 1,000 ng dosage, there is an inhibition of the physiologic angiogenesis with a higher area of non-perfused retina.

(c) 2020 S. Karger AG, Basel

\section{Introduction}

Retinopathy of prematurity (ROP) in premature infants has been reported to place approximately 20,000 infants at risk of becoming blind annually on a global scale.

Sarina M. Amin and Andres Gonzales contributed equally to this work. karger@karger.com

www.karger.com/ore

Karger $\stackrel{2}{*}$
(C) 2020 S. Karger AG, Basel
Swati Agarwal-Sinha Associate Professor

Department of Ophthalmology, Director, Retinopathy of Prematurity Services University of Florida, 1600 SW Archer Road

Gainesville FL 32608 (USA)

sxapublish@gmail.com 
ROP is even more threatening in South Asian and African countries due to higher numbers of preterm births coupled with inadequate neonatal care [1]. Medical care to prevent blindness secondary to ROP has gained attention globally in the last decade with the increased use of antivascular endothelial growth factor (anti-VEGF) intravitreal injections (bevacizumab, ranibizumab, and aflibercept) as the primary mode of treatment for type $1 \mathrm{ROP}$ over traditional laser photocoagulation to the avascular retina [2]. There are numerous clinical studies which highlight the efficacy of anti-VEGF injections in the treatment of ROP [3-6]. Although bevacizumab is used widely in the treatment of type 1 ROP due to its low cost compared with other anti-VEGF medications, there are published studies on the efficacy of ranibizumab and aflibercept in neonates with promising results [4-9]. Aflibercept use in neonates for the treatment of ROP has been reported outside of the USA [5, 7-9]. Aflibercept use in the treatment of ROP has not been widely adopted in the USA and other developed countries; however, it is commonly used in the management of retinal vascular diseases, such as neovascular age-related macular degeneration, diabetic retinopathy, macular edema, and retinal vein occlusion.

The pathogenesis of ROP is multifactorial. Prematurity, low birth weight, supplemental oxygen demand, and fluctuations in oxygenation are implicated as risk factors for the development and progression of ROP [10-12]. Chemical mediators that have been implicated in the pathogenesis of angiogenesis include VEGF-A, placental growth factor (PlGF), erythropoietin, insulin-like growth factor, and others. VEGF-A and PlGF have been implicated to have a potential role in neovascularization [1316]. In recent years, studies have implicated intrauterine inflammation, chronic placental inflammation, oxidative stress, and generalized inflammation as major risk factors in the pathologic angiogenesis and development and progression of ROP [17-22].

There are limited published animal studies with the use of aflibercept in the oxygen-induced retinopathy (OIR) models. Canine OIR models have found regression of neovascularization at both higher and lower doses of aflibercept, although with inhibition of normal retinal vascularization at higher doses [23]. Regressed neovascularization with an increase in avascular retina was also found in a mouse OIR model, along with corresponding decrease in electroretinographic amplitudes [24]. Mouse OIR models evaluating the effect of PlGF modulation (sunitinib) and/or VEGF blockade (aflibercept) found inhibition of neovascularization in both groups, with the
Table 1. Number of mouse eyes used in each study group

\begin{tabular}{|c|c|c|c|c|c|}
\hline \multirow{2}{*}{$\begin{array}{l}\text { Postnatal } \\
\text { age }\end{array}$} & \multirow{2}{*}{$\begin{array}{l}\text { Days post- } \\
\text { injection }\end{array}$} & \multirow[t]{2}{*}{ Normoxia } & \multicolumn{3}{|l|}{ Hyperoxia } \\
\hline & & & $\begin{array}{l}0 \mathrm{ng} \\
\text { aflibercept }\end{array}$ & $\begin{array}{l}100 \mathrm{ng} \\
\text { aflibercept }\end{array}$ & $\begin{array}{l}1,000 \mathrm{ng} \\
\text { aflibercept }\end{array}$ \\
\hline P17 & 3 & 11 & 9 & 25 & 16 \\
\hline P25 & 11 & 10 & 5 & 10 & 19 \\
\hline
\end{tabular}

PlGF-inhibited eyes showing impaired physiologic angiogenesis [25].

In the OIR animal studies discussed previously [23, 24], a relatively high dose of aflibercept was used to study the regression of neovascularization, and persistent changes in the retinal structures posttreatment were noted. The purpose of this study was to determine if a lower dose of aflibercept could be utilized that would provide reduction in areas of non-perfused retina, while simultaneously preserving normal vascular angiogenesis in the OIR mouse model of ROP.

\section{Methods}

Animals

All experiments using animals were approved by the Institutional Animal Care and Use Committee at the University of Florida and conducted in accordance with the ARVO Statement for the Use of Animals in Ophthalmic and Vision Research and National Institutes of Health regulations.

OIR Induction and Normoxic Control

C57BL/6J mice were obtained from the University of Florida Animal Care Service and maintained under standard laboratory conditions $\left(18-23^{\circ} \mathrm{C}, 40-50 \%\right.$ humidity) with food and water available ad libitum. To generate the OIR, we followed the procedures of Stahl et al. [26] Briefly, the pups were placed in a hyperoxic plexiglass container at postnatal day 7 with the dams (mothers). The oxygen content was maintained at $75 \pm 2 \%$ oxygen using a calibrated regulator/sensor (Proox Model 110, BioSpherix). After 5 days in the hyperoxic conditions, the mice were returned to room air at postnatal day 12 and maintained in this environment until the end of the protocol. In parallel, a set of normoxic mice were maintained in room air for the duration of the protocol.

\section{Group Assignments}

The mice were grouped as PN17 ( $n=61$ eyes) and PN25 $(n=$ 44 eyes) and thereafter randomly assigned to normoxic ( $n=21$ eyes) or hyperoxic conditions ( $n=84$ eyes). Those mice exposed to hyperoxia ( $n=84$ eyes) were randomly assigned to 1 of 3 treatment groups receiving $0 \mathrm{ng}(n=14$ eyes), $100 \mathrm{ng}(n=35$ eyes), or $1,000 \mathrm{ng}(n=35$ eyes) of aflibercept (Table 1). Groups were unequal due to inherent mice pup losses sustained due to various fac-
92

Ophthalmic Res 2021;64:91-98

DOI: $10.1159 / 000509380$
Amin/Gonzalez/Guevara/Bolch/ Andersen/Smith/Agarwal-Sinha 


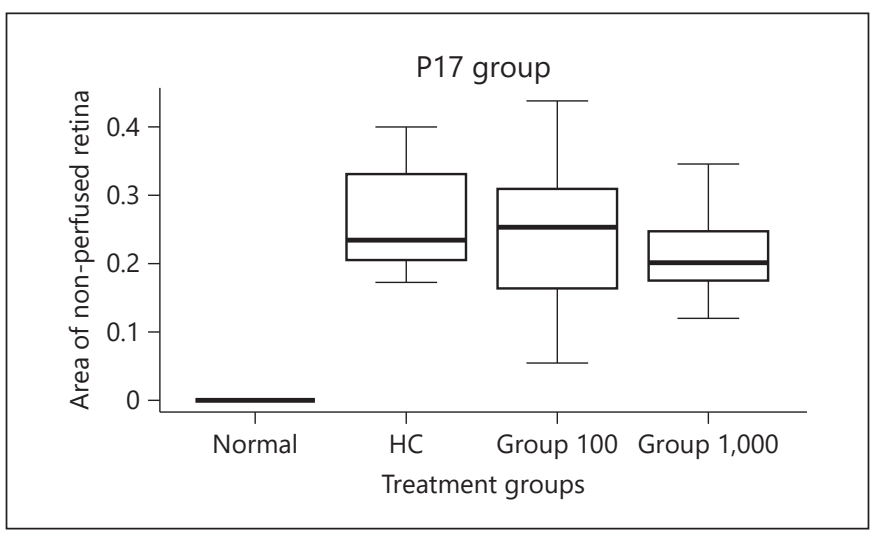

Fig. 1. Comparison of areas of non-perfusion in hyperoxic mice treated for 3 days with aflibercept. Areas of non-perfusion were measured at PN17 (3 days post-aflibercept treatment) in mice maintained in normal room air (normal), mice exposed to hyperoxia (HC), mice exposed to hyperoxia and treated with $100 \mathrm{ng}$ aflibercept (group 100), and mice exposed to hyperoxia and treated with 1,000 ng aflibercept (group 1,000). At 3 days posttreatment, there was no significant difference in retinal non-perfusion between the hyperoxic control and treatment groups. HC, hyperoxic control.

tors. The aliquots of aflibercept were combined with sterile PBS to equal a standardized $1 \mu \mathrm{L}$ for each injection. The dosage range of aflibercept administered was based on the canine study in which an effective dose of 25-250 $\mu \mathrm{g}$ for VEGF Trap was determined [23]. We then used the vitreous volume ratio of the canine to mouse eye of $1.7 \mathrm{~mL}$ for the dog eye [27] and $5.3 \mu \mathrm{L}$ for the mouse eye [28, 29 ], determining that an equivalent dose in mouse would be $78-$ $780 \mathrm{ng}$.

\section{Intravitreal Injection Procedure}

Two days after returning to room air (day 14, PN14), mice were anesthetized with ketamine $(72 \mathrm{mg} / \mathrm{kg})$ and xylazine $(4 \mathrm{mg} / \mathrm{kg})$, and $1 \mu \mathrm{L}$ intravitreal injections of aflibercept dilutions administered. Neomycin/polymyxin B/dexamethasone ophthalmic ointment (Bausch \& Lomb Inc.) was applied after injection. The mice were recovered from anesthesia and returned to their housing under room air.

\section{Retinal Preparation}

At day 3 (PN17) and day 11 (PN25) postinjection, the mice were euthanized, enucleated, and retinal flat mounts prepared. Enucleated eyes were fixed in $4 \%$ paraformaldehyde in PBS for 60 min and then placed in PBS for storage at $4^{\circ} \mathrm{C}$. Mouse retinal cups were carefully dissected and placed in PBS. Retinal cups were then permeabilized with $0.3 \%$ Triton X-100 in PBS with 5\% low Ig fetal bovine serum (ThermoFisher) and $0.2 \%$ bovine serum albumin (Sigma) to block the tissue overnight at $4^{\circ} \mathrm{C}$. After the removal of the blocking solution, the retinal vasculature was stained with anti-collagen IV antibody (AB756P; Chemicon, Temecula, CA, USA), diluted $1: 200$ in PBS with $0.1 \%$ TritonX-100, $0.2 \%$ bovine serum albumin, and $1 \%$ low IgG fetal bovine serum, incubating overnight at $4^{\circ} \mathrm{C}$. The primary antibody was removed, and retinal

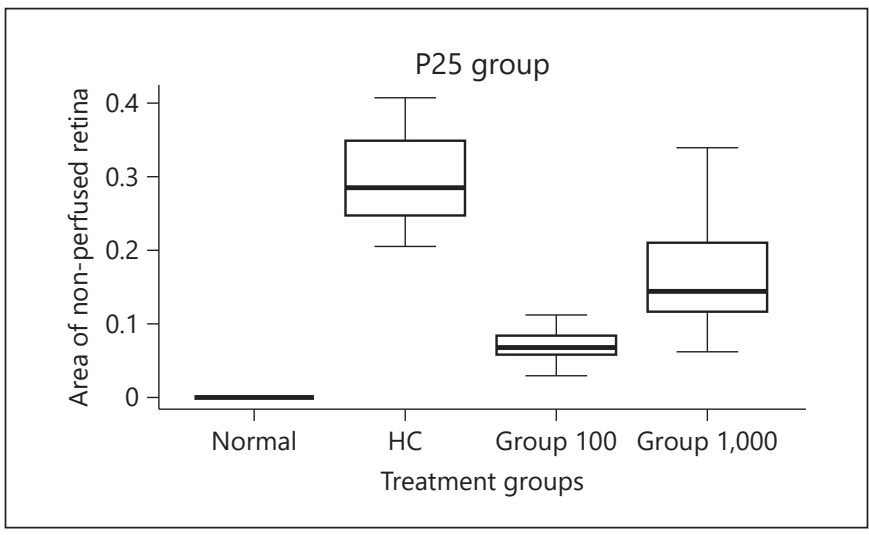

Fig. 2. Areas of retinal non-perfusion are reduced in mice 11 days (PN25) after treatment with aflibercept. At PN25, there was a significant difference between $\mathrm{HC}$ and the $100 \mathrm{ng}$ treatment group, with reduction in retinal non-perfusion in the treated group. Interestingly, there is a higher area of retinal non-perfusion seen in the 1,000 ng treatment group, possibly indicating secondary toxicity to the development of normal retinal vasculature at the higher dose. HC, hyperoxic control. tissues were washed with PBS twice prior to instillation of secondary antibody consisting of 1:600 anti-rabbit-Alexa Fluor 488 (ThermoFisher) diluted in PBS with $0.1 \%$ Triton X-100, $0.2 \%$ bovine serum albumin, and $1 \%$ low Ig fetal bovine serum with DAPI $(10 \mu \mathrm{g} / \mathrm{mL})$ to stain nuclei. After incubating overnight $\left(4^{\circ} \mathrm{C}\right)$, the retinal tissues were rinsed 3 times with $\mathrm{PBS}$ and retinal flat mounts prepared for imaging.

\section{Flat Mount Analysis}

Flat mount preparations were imaged with fluorescence microscopy (BZ-9000, Keyence Corp), and fields of view automatically stitched together to form an image of the entire retinal flat mount (BZ-II Analyzer software, Keyence Corp). The flat mount images were processed for analysis in ImageJ software. Blinded clinicians manually outlined areas of vascularized retina and subsequently manually demarcated of areas of non-perfusion. The surface areas of both vascularized retina and avascular retina were calculated for each sample.

\section{Statistical Analysis}

For each retina, the non-perfused area values were normalized by dividing the area of non-perfusion by the total retinal area to normalize for variations in retinal size and for any experimental losses during the preparation of the retinal flat mounts. An ANOVA was conducted in order to determine if there was a difference in the fraction of non-perfused retinal area between the temporally independent groups (PN17 and PN25) and the treatment groups (Normal, HC, $100 \mathrm{ng}$, and 1,000 ng). Tukey's method for multiple comparisons of means with $95 \%$ confidence intervals was then conducted to discern where the possible differences between the groups and treatments were located. The statistical significance level was $p$ value $<0.05$. All statistical analyses were run using $\mathrm{R}$ (version 3.5.1). 


\section{Results}

Post-injection Day 3 (PN17)

Our data show that on postinjection day 3 (PN17 group), there was no significant difference in the area of non-perfused retina between the hyperoxic control and both the 100 and 1,000 ng aflibercept treated groups (Fig. 1; Table 2). There was a significant difference in the non-perfusion status among normal retinas $(p<0.05)$ when compared to the hyperoxic controls and both 100 and 1,000 ng treatment groups, with normal retinas displaying decreased non-perfusion compared to all other groups exposed to hyperoxia (Fig. 1,3). These results indicate that hyperoxic treatment leads to increased retinal non-perfusion as expected and that there is a lack of sig- nificant treatment effect of the 100 and 1,000 ng aflibercept doses at 3 days postinjection.

\section{Post-injection Day 11 (PN25)}

On postinjection day 11 (PN25 group), there was a significant difference in the area of non-perfused retina between the hyperoxic control and the groups treated with 100 and 1,000 ng aflibercept, with the treatment groups displaying smaller areas of retinal non-perfusion. There was also a significant difference when comparing the 1,000 and $100 \mathrm{ng}$ aflibercept treatment groups, with the $1,000 \mathrm{ng}$ group displaying an increased area of non-perfusion (Fig. 2; Table 2). This observation indicates that differences in the non-perfusion status become evident between the control and aflibercept treatment groups by PN25, which

Table 2. Results comparing the PN17 groups, PN25 groups, and PN25 versus PN17 groups

\begin{tabular}{llrrl}
\hline & Diff & Lwr & Upr & $p$ adj \\
\hline P17 group & & & & \\
Normal-HC & $-2.624813 \mathrm{e}^{-1}$ & 0.361830061 & 0.163132605 & 0.0000000 \\
Normal-100 & $-2.455083 \mathrm{e}^{-1}$ & -0.325482443 & -0.165534076 & 0.0000000 \\
Normal-1,000 & $-2.110819 \mathrm{e}^{-1}$ & -0.297656528 & -0.124507341 & 0.0000000 \\
HC-100 & $1.697307 \mathrm{e}^{-2}$ & -0.068950655 & 0.102896801 & 0.9986448 \\
HC-1,000 & $5.139940 \mathrm{e}^{-2}$ & -0.040699338 & 0.143498134 & 0.6684689 \\
1,000-100 & $-3.442633 \mathrm{e}^{-2}$ & -0.105192683 & 0.036340033 & 0.8018192 \\
\hline P25 group & & & & \\
Normal-HC & $-2.979435 \mathrm{e}^{-1}$ & -0.419010417 & -0.176876552 & 0.0000000 \\
Normal-100 & $-7.045158 \mathrm{e}^{-2}$ & -0.169302313 & 0.028399160 & 0.3562593 \\
Normal-1,000 & $-1.651488 \mathrm{e}^{-1}$ & -0.251503752 & -0.078793902 & $\mathbf{0 . 0 0 0 0 0 1 3}$ \\
HC-100 & $2.274919 \mathrm{e}^{-1}$ & 0.106424975 & 0.348558840 & $\mathbf{0 . 0 0 0 0 0 2 1}$ \\
HC-1,000 & $1.327947 \mathrm{e}^{-1}$ & 0.021696021 & 0.243893294 & $\mathbf{0 . 0 0 8 1 8 3 7}$ \\
1,000-100 & $9.469725 \mathrm{e}^{-2}$ & 0.008342326 & 0.181052175 & $\mathbf{0 . 0 2 1 2 8 1 0}$ \\
\hline P25 versus P17 & & & & \\
P25 100-P17 100 & $-1.750567 \mathrm{e}^{-1}$ & -0.257761143 & -0.092352223 & $\mathbf{0 . 0 0 0 0 0 0 1}$ \\
P25 1,000-P17 1,000 & $-4.593311 \mathrm{e}^{-2}$ & -0.120933259 & 0.029067044 & 0.5560545 \\
P25 100-P17 1,000 & $-1.406304 \mathrm{e}^{-1}$ & -0.229733208 & -0.051527508 & $\mathbf{0 . 0 0 0 1 0 6 1}$ \\
P25 1,000-P17 100 & $-8.035943 \mathrm{e}^{-2}$ & -0.147632998 & -0.013085867 & $\mathbf{0 . 0 0 8 2 4 7 3}$ \\
\hline
\end{tabular}

Diff, difference in the values of the fraction of area non-perfusion; lwr, lower values for the $95 \%$ confidence interval for the mean difference; upr, upper values for the $95 \%$ confidence interval for the mean difference; $p$ adj: $p$ value for the $t$ test comparing those two groups. Statistically significant $p$ value $<0.05$.

Fig. 3. a Flat mounts of isolated mouse retinas highlighting the retinal vasculature by staining for collagen IV. a Flat mount of a mice retina housed under room air. $\mathbf{b}$ Flat mount of the retina of a mouse housed in 75\% oxygen (hyperoxic control group), with areas of central non-perfused retina (white arrow) and multiple areas of neovascularization along the vascular-avascular junctions (red circles). c Flat mount of a mouse retina from the PN17 group treated with $100 \mathrm{ng}$ aflibercept showing decreased areas of non-per- fused retina. $\mathbf{d}$ Flat mount of a mouse retina from the PN25 group treated with $100 \mathrm{ng}$ aflibercept showing significant decrease in areas of non-perfused retina. e Flat mount of a mouse retina from the PN17 group treated with 1,000 ng aflibercept showing decreased areas of neovascularization with non-perfused retina. $f$ Flat mount of a mouse retina from the PN25 group treated with 1,000 ng aflibercept showing significant decrease in areas of non-perfused retina.

(For figure see next page.)

94

Ophthalmic Res 2021;64:91-98

DOI: $10.1159 / 000509380$
Amin/Gonzalez/Guevara/Bolch/ Andersen/Smith/Agarwal-Sinha 

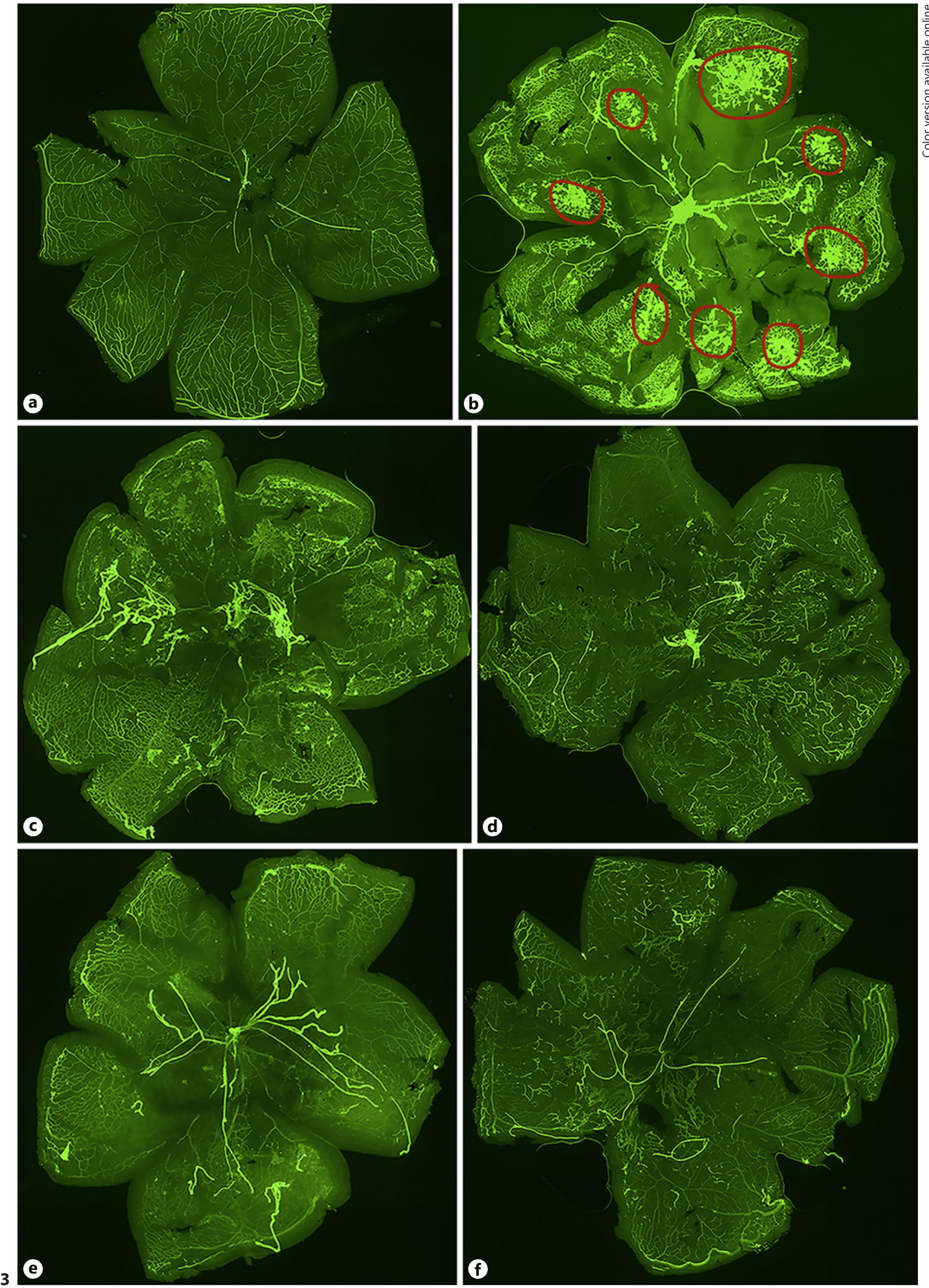
were not noted by PN17. This suggests that significant treatment effect occurs between day 3 and day 11 postinjection. With respect to the larger amount of non-perfusion in the 1,000 ng group, it may indicate a possible inhibition of vascular development at higher doses.

\section{Comparing PN17 and PN25 Groups}

For the $100 \mathrm{ng}$ aflibercept treatment group, there was a statistically significant decrease in retinal non-perfusion at PN25 when compared to PN17. Again, this highlights increased treatment effect at day 11 versus day 3 postinjection. There was no statistically significant difference in the non-perfusion status for the 1,000 ng aflibercepttreated groups between these time points. These results indicate that there appears to be significant vascular recovery at the PN25 time point with the $100 \mathrm{ng}$ aflibercept dose, with no significant vascular recovery observed in the 1,000 ng group (Fig. 3; Table 2).

\section{Discussion}

VEGF plays a vital role in normal physiologic and pathologic retinal angiogenesis, with inhibition of VEGF resulting in the reduction of retinal non-perfusion and consequent neovascularization. There are a limited number of animal studies in the literature that have evaluated the effects of aflibercept on retinal vasculature exposed to a hyperoxic state. These studies noted that although aflibercept promoted neovascular tuft regression, higher doses inhibited normal retinal revascularization and potentially led to permanent changes in neuroretinal structures [23-25]. It has been postulated that this is secondary to differences in binding of vitreous VEGF and retinal VEGF, which is a factor in normal retinal angiogenesis. However, prior studies have shown that in subjects with expected low levels of vitreous VEGF, aflibercept injection did not affect normal retinal angiogenesis; therefore, other factors are likely playing a role in normal angiogenesis. It is reported that VEGF is a more significant driver of pathologic angiogenesis than physiologic angiogenesis; thus, VEGF blockade with aflibercept mostly affects pathologic neovascularization, while sparing normal retinal vasculature [23].

Given the importance of dosage effects on retinal vasculature established by Lutty et al. [23], the present study focused on the differences in retinal non-perfusion at varying doses of aflibercept in the mouse OIR model. The area of non-perfusion was studied, as it is the driving factor for VEGF production and consequent neovascular- ization. Our study demonstrated that treatment with aflibercept can reduce non-perfusion of retinas exposed to hyperoxia in the mouse model of OIR, although treatment effects may not be observed until 11 days posttreatment (PN25 group). A significant reduction of retinal non-perfusion was noted in the PN25 group at both 100 and 1,000 ng aflibercept doses. However, it was the 100 ng aflibercept treatment group at day 11 postinjection which displayed vascular recovery and preserved physiologic angiogenesis when compared to the 100 and 1,000 ng treated groups at day 3 postinjection. The higher dose $1,000 \mathrm{ng}$ aflibercept treatment group did not show a significant difference in non-perfusion from day 3 to day 11 postinjection. This could be due to higher retinal VEGF binding with aflibercept due to the saturation of vitreous VEGF binding at significantly higher doses, with our higher treatment dose being ten times more concentrated than our lower treatment dose. However, studies on normal retinas of nonhuman primates did not show adverse effects on retinal vasculature morphology or ERG function with repeated aflibercept treatment [30]. These varying results could be secondary to different equivalent dosages administered or difference in animal models, in addition to the different factors playing a role in physiologic retinal angiogenesis.

This relationship between retinal vascular status and aflibercept dosage at different time points (PN17 and PN25) highlights the importance of short-term and longterm evaluation of aflibercept treatment in ROP models. It is important to consider this temporal difference in order to determine potential detrimental outcomes on the retinal vasculature, such as inhibition of normal retinal vascularization. Our data suggest that a precise titration of aflibercept dose can result in a balance of regression of pathologic non-perfusion with normal retinal vascular recovery if the short-term and long-term effects of aflibercept on retinal vasculature are assessed. In this study, 100 ng of aflibercept appears to be an effective dose that balances these 2 factors and may potentially be extrapolated to an ROP setting. Based on the results of our study, we determined the extrapolated dosage of aflibercept for a preterm infant (defined as an infant born prior to 37 weeks gestation) to be $0.0147-0.1472 \mathrm{mg}$ with an average volume of injection of $0.02 \mathrm{~mL}$ of $2 \mathrm{mg} / 0.5 \mathrm{~mL}$ aflibercept. This is based on an estimated vitreous cavity volume of 1 $\mathrm{mL}$ in the preterm infant [31]. The range of VEGF Trap dose calculated was $0.004-0.037 \mathrm{~mL}$, with the smaller doses presumably still maintaining efficacy in eyes with smaller vitreous volumes. The vitreous cavity volumes of premature infants prior to 32 weeks gestation have not
96

Ophthalmic Res 2021;64:91-98 DOI: $10.1159 / 000509380$
Amin/Gonzalez/Guevara/Bolch/ Andersen/Smith/Agarwal-Sinha 
been documented in the literature, so extrapolated doses cannot be applied to this subset of patients. This dosage is an approximation, as vitreous volumes can differ significantly depending on gestational age. Long-term evaluation of this dose would need to be further assessed in animal studies prior to use in human neonates.

Limitations of our study stem from objective quantification of non-perfused areas of retina, as this required manual delineation. In order to control for this and reduce data collection bias, all non-perfused areas were manually outlined by the same clinician investigator to eliminate variation of analysis between investigators. Our study also did not quantify areas of neovascularization. We believe that non-perfusion is the primary driver of VEGF-mediated neovascularization, and therefore, retinal non-perfusion was chosen as the end point for analysis. In addition, OIR mouse models of ROP can undergo spontaneous regression of neovascularization between $\mathrm{PN} 17$ and PN25 [32], which would have confounded our treatment effect.

\section{Conclusion}

This study evaluated the effective dosage and longterm effects of aflibercept on retinal perfusion using the OIR mouse model of ROP and determined that aflibercept treatment resulted in improved retinal perfusion. However, treatment effects were more apparent at day 11 posttreatment. A dose of $100 \mathrm{ng}$ in our study led to vascular recovery without inhibition of physiologic angiogenesis at 11 days. A higher dose of aflibercept (1,000 ng) inhibited retinal vascular recovery at day 11 posttreatment. These results suggest that in addition to precise titration of antiVEGF dose to balance inhibition of neovascularization with retinal non-perfusion, longer observation after antiVEGF treatment is required to evaluate its effect on vascular recovery and physiologic angiogenesis.

\section{Acknowledgement}

The authors thank the UF animal research laboratory team for assistance with housing and care of mice.

\section{Statement of Ethics}

All experiments using animals were approved by the Institutional Animal Care and Use Committee at the University of Florida and conducted in accordance with the ARVO Statement for the Use of Animals in Ophthalmic and Vision Research and National Institutes of Health regulations.

\section{Conflicts of Interest Statement}

The authors have no conflicts of interest to declare.

\section{Funding Sources}

This study was funded by an unrestricted grant from Research to Prevent Blindness to the University of Florida.

\section{Author Contributions}

Sarina Amin, MD, contributed to literature search, figures, data collection, data analysis, data interpretation, writing, and editing. Andres Gonazalez, MD, contributed to literature search, data collection, data analysis, data interpretation, writing, and editing. Jade Guevara, MD, contributed to data collection, data analysis, and data interpretation. Charlotte Bolch, $\mathrm{PhD}$, contributed in the statistical analysis of the results and production of box plot figures. Lorick Andersen, MD, contributed to data collection, data analysis, and data interpretation. Clay Smith, $\mathrm{PhD}$, contributed to study design, literature search, data collection, data analysis, data interpretation, editing, and final approval of manuscript. Swati Agarwal-Sinha, MD, contributed to study design, literature search, data collection, data interpretation, writing, editing, and final approval of manuscript.

\section{References}

1 Gilbert C, Blencowe H; Community Eye Health. Retinopathy of prematurity: it is time to take action. Community Eye Health. 2017; 30(99):45-8.

2 Agarwal-Sinha S, Amin S, Way A. Preferences and trends in practices caring premature infants for retinopathy of prematurity (ROP): a web-based survey. Curr Trends Ophthalmol. 2018;1(1):49-61.
3 Mintz-Hittner HA, Kuffel RR Jr. Intravitreal injection of bevacizumab (avastin) for treatment of stage 3 retinopathy of prematurity in zone I or posterior zone II. Retina. 2008;28(6): 831-8.

4 Kang HG, Choi EY, Byeon SH, Kim SS, Koh $\mathrm{HJ}$, Lee SC, et al. Intravitreal ranibizumab versus laser photocoagulation for retinopathy of prematurity: efficacy, anatomical outcomes and safety. Br J Ophthalmol. 2019;103(9): $1332-6$.
5 Sukgen EA, Koçluk Y. Comparison of clinical outcomes of intravitreal ranibizumab and aflibercept treatment for retinopathy of prematurity. Graefes Arch Clin Exp Ophthalmol. 2019;257(1):49-55.

6 Kimyon S, Mete A. Comparison of bevacizumab and ranibizumab in the treatment of type 1 retinopathy of prematurity affecting zone 1. Ophthalmologica. 2018;240(2):99105 . 
7 Ekinci DY, Çelik K. Comparison of the efficacy between intravitreal aflibercept and laser photocoagulation in the treatment of retinopathy of prematurity. J Pediatr Ophthalmol Strabismus. 2020;57(1):54-60.

8 Vedantham V. Intravitreal aflibercept injection in Indian eyes with retinopathy of prematurity. Indian J Ophthalmol. 2019;67(6): 884-8.

9 Vural A, Perente İ, Onur İU, Eriş E, Seymen Z, Hergünsel GO, et al. Efficacy of intravitreal aflibercept monotherapy in retinopathy of prematurity evaluated by periodic fluorescence angiography and optical coherence tomography. Int Ophthalmol. 2019;39(10): 2161-9.

10 Patz A. The effect of oxygen on immature retinal vessels. Invest Ophthalmol. 1965;4(6): 988-99.

11 McColm JR, Cunningham S, Wade J, Sedowofia K, Gellen B, Sharma T, et al. Hypoxic oxygen fluctuations produce less severe retinopathy than hyperoxic fluctuations in a rat model of retinopathy of prematurity. Pediatr Res. 2004;55(1):107-13.

12 The STOP-ROP Multicenter Study Group Supplemental therapeutic oxygen for prethreshold retinopathy of prematurity (STOP$\mathrm{ROP}$ ), a randomized controlled trial. I: primary outcomes. Pediatrics. 2000;105:295310.

13 Smith LE, Kopchick JJ, Chen W, Knapp J, Kinose F, Daley D, et al. Essential role of growth hormone in ischemia-induced retinal neovascularization. Science. 1997;276(5319):17069.

14 Smith LE, Shen W, Perruzzi C, Soker S, Kinose F, Xu X, et al. Regulation of vascular endothelial growth factor-dependent retinal neovascularization by insulin-like growth factor-1 receptor. Nat Med. 1999;5(12):13905.
15 Morita M, Ohneda O, Yamashita T, Takahashi S, Suzuki N, Nakajima O, et al. HLF/ HIF-2alpha is a key factor in retinopathy of prematurity in association with erythropoietin. EMBO J. 2003;22(5):1134-46.

16 Zhou AY, Bai YJ, Zhao M, Yu WZ, Huang LZ, $\mathrm{Li}$ XX. Placental growth factor expression is reversed by antivascular endothelial growth factor therapy under hypoxic conditions. World J Pediatr. 2014;10(3):262-70.

17 Pietrasanta C, Pugni L, Merlo D, Acaia B, Consonni D, Ronchi A, et al. Impact of different stages of intrauterine inflammation on outcome of preterm neonates: gestational age-dependent and -independent effect. PLoS One. 2019;14(2):e0211484.

18 Aouiss A, Anka Idrissi D, Kabine M, Zaid Y. Update of inflammatory proliferative retinopathy: ischemia, hypoxia and angiogenesis. Curr Res Transl Med. 2019;67(2):62-71

19 Kim CY, Jung E, Kim EN, Kim CJ, Lee JY, Hwang JH, et al. Chronic placental inflammation as a risk factor of severe retinopathy of prematurity. J Pathol Transl Med. 2018;52(5): 290-7.

20 Lynch AM, Berning AA, Thevarajah TS, Wagner BD, Post MD, McCourt EA, et al. The role of the maternal and fetal inflammatory response in retinopathy of prematurity. Am J Reprod Immunol. 2018;80(3):e12986.

21 Rivera JC, Dabouz R, Noueihed B, Omri S, Tahiri H, Chemtob S. Ischemic retinopathies: oxidative stress and inflammation. Oxid Med Cell Longev. 2017;2017:3940241.

22 Rivera JC, Holm M, Austeng D, Morken TS, Zhou TE, Beaudry-Richard A, et al. Retinopathy of prematurity: inflammation, choroidal degeneration, and novel promising therapeutic strategies. J Neuroinflammation. 2017; 14(1):165
23 Lutty GA, McLeod DS, Bhutto I, Wiegand SJ. Effect of VEGF trap on normal retinal vascular development and oxygen-induced retinopathy in the dog. Invest Ophthalmol Vis Sci. 2011;52(7):4039-47.

24 Tokunaga CC, Mitton KP, Dailey W, Massoll C, Roumayah K, Guzman E, et al. Effects of anti-VEGF treatment on the recovery of the developing retina following oxygen-induced retinopathy. Invest Ophthalmol Vis Sci. 2014; 55(3):1884-92.

25 Heiduschka P, Plagemann T, Li L, Alex AF, Eter N. Different effects of various anti-angiogenic treatments in an experimental mouse model of retinopathy of prematurity. Clin Experiment Ophthalmol. 2019;47(1):79-87.

26 Stahl A, Connor KM, Sapieha P, Chen J, Dennison RJ, Krah NM, et al. The mouse retina as an angiogenesis model. Invest Ophthalmol Vis Sci. 2010;51(6):2813-26.

27 Gilger BC, Reeves KA, Salmon JH. Ocular parameters related to drug delivery in the canine and equine eye: aqueous and vitreous humor volume and scleral surface area and thickness. Vet Ophthalmol. 2005;8:265-9.

28 Remtulla S, Hallett PE. A schematic eye for the mouse, and comparisons with the rat. $\mathrm{Vi}$ sion Res. 1985;25(1):21-31.

29 Jeon CJ, Strettoi E, Masland RH. The major cell populations of the mouse retina. J Neurosci. 1998;18(21):8936-46.

30 Zimmer E, et al. IOVS. 2006;47.ARVO E-Abstract 1751

31 Harnett ME. Pathophysiology and mechanisms of severe retinopathy of prematurity. Ophthalmology. 2015;122(1):200-10.

32 Connor KM, Krah NM, Dennison RJ, Aderman CM, Chen J, Guerin KI, et al. Quantification of oxygen-induced retinopathy in the mouse: a model of vessel loss, vessel regrowth and pathological angiogenesis. Nat Protoc. 2009;4(11):1565-73. 